\title{
Auroral evidence of a localized magnetic anomaly in Jupiter's northern hemisphere
}

\author{
Denis Grodent, ${ }^{1}$ Bertrand Bonfond, ${ }^{1}$ Jean-Claude Gérard, ${ }^{1}$ Aikaterini Radioti, ${ }^{1}$ \\ Jacques Gustin, ${ }^{1}$ John T. Clarke, ${ }^{2}$ Jonathan Nichols, ${ }^{2}$ and John E. P. Connerney ${ }^{3}$ \\ Received 19 March 2008; revised 30 May 2008; accepted 6 June 2008; published 3 September 2008.
}

[1] We analyze more than 1000 HST/Advanced Camera for Survey images of the ultraviolet auroral emissions appearing in the northern hemisphere of Jupiter. The auroral footprints of Io, Europa, and Ganymede form individual footpaths, which are fitted with three reference contours. The satellite footprints provide a convenient mapping between the northern Jovian ionosphere and the equatorial plane in the middle magnetosphere, independent of any magnetic field model. The VIP4 magnetic field model is in relatively good agreement with the observed footprint of Io. However, in the auroral kink sector, between the $80^{\circ}$ and $150^{\circ}$ System III meridians, the model significantly departs from the observation. One possible way to improve the agreement between the VIP4 model and the observed footprints is to include a magnetic anomaly. We suggest that this anomaly is characterized by a weakening of the surface magnetic field in the kink sector and by an added localized tilted dipole field. This dipole rotates with the planet at a depth of $0.245 R_{J}$ below the surface, and its magnitude is set to $\sim 1 \%$ of Jupiter's dipole moment. The anomaly has a very limited influence on the magnetic field intensity in the equatorial plane between the orbits of Io and Ganymede. However, it is sufficient to bend the field lines near the high-latitude atmosphere and to reproduce the observed satellite ultraviolet footpaths. JUNO's in situ measurements will determine the structure of Jupiter's magnetic field in detail to expand on these results.

Citation: Grodent, D., B. Bonfond, J.-C. Gérard, A. Radioti, J. Gustin, J. T. Clarke, J. Nichols, and J. E. P. Connerney (2008), Auroral evidence of a localized magnetic anomaly in Jupiter's northern hemisphere, J. Geophys. Res., 113, A09201, doi:10.1029/2008JA013185.

\section{Introduction}

[2] The magnetic field of Jupiter and the plasma sheet flowing near its equatorial plane undergo a permanent electromagnetic interaction with Jupiter's moons Io, Europa and Ganymede. The orbital velocity of the moons is smaller than the fast rotation of the magnetic field which is nearrigidly spinning with the planet. In a first approximation, the resulting differential motion of the moons, which are conducting bodies, in the magnetic field gives rise to current systems along the magnetic field lines connecting the moons to the ionosphere of Jupiter. The current carriers, mostly electrons, interact with Jupiter's ionosphere. When they precipitate in the atmosphere they collide with the neutrals $\left(\mathrm{H}_{2}, \mathrm{H}\right)$ and lose their energy which is converted into heat, ionization, and auroral emissions. Accordingly, each of the three moons leaves an isolated auroral footprint around the poles of Jupiter [e.g., Clarke et al., 2002]. So far, it has not been possible to discriminate the footprint of

\footnotetext{
${ }^{1}$ Laboratory for Planetary and Atmospheric Physics, Université de Liège, Liège, Belgium. USA.

${ }^{2}$ Center for Space Physics, Boston University, Boston, Massachusetts,

${ }^{3}$ NASA Goddard Space Flight Center, Greenbelt, Maryland, USA.
}

Copyright 2008 by the American Geophysical Union. 0148-0227/08/2008JA013185\$09.00
Callisto from the main auroral emission. Their location is mainly controlled by the topology of the field lines. The analysis of the auroral footprints thus provides information on the magnetic field itself.

[3] Reality is obviously much more complex than this simplified view. As an example, it has recently been shown that the ultraviolet (UV) auroral footprint of Io is not a unique circular spot with constant brightness [Gérard et al., 2006; Bonfond et al., 2007a, 2008]. High-resolution HST images reveal that the footprint consists of multiple spots whose location, size, inter-distance and brightness are controlled, or at least influenced, by the position of Io in the Io plasma torus. This plasma reservoir is tapped with ions resulting from the intense volcanic activity of Io. It is shaped by the combined effect of centrifugal force, density pressure gradient, and magnetic trapping. The magnetic field forces the plasma in the torus to corotate with the planet. Ultimately, the iogenic plasma escapes the torus and populates the middle and outer magnetosphere. During its radial journey through the equatorial plane, the angular momentum acquired by the plasma in the torus is conserved. As it moves away from Jupiter, its angular velocity decreases and a plasma angular velocity gradient is set up. Field aligned currents are associated with this corotation breakdown and give rise to strong auroral emissions [e.g., Hill, 2001; Cowley and Bunce, 2001; Southwood and 
Kivelson, 2001]. These field-aligned or Birkeland currents are broadly distributed within the middle magnetosphere current sheet, they become significant from distances of order of $\sim 20$ Jovian radii $\left(\mathrm{R}_{\mathrm{J}}, 1 \mathrm{R}_{\mathrm{J}}=71323 \mathrm{~km}\right)$ and thus their associated auroral signature appears poleward of the satellite footprints. This emission is usually referred to as the main auroral oval however, following Grodent et al. [2008] we prefer to refer to this emission as the main auroral emission (ME) rather than the main oval, since it is clearly not an oval, not even a closed contour. Its morphology has been widely studied [e.g., Gérard et al., 1993; Prangé et al., 1997; Clarke et al., 1998, 2004; Grodent et al., 2003] and it was shown that the general shape of the main emission remains roughly constant, even over time periods spanning several years. The relative stability of the ME then made it possible to define an average contour giving its most likely location.

[4] The satellite footpaths are particularly useful because the satellites are orbiting on quasi-circular paths. As a result, one auroral footprint position (latitude-longitude) can be associated with one orbital longitude and radius and may be used to constrain magnetic field models. The case of the ME is less obvious since Galileo and other spacecraft have shown that local time effects influence the plasma velocity distribution around Jupiter [Krupp et al., 2001; Khurana, 2001]. The distance at which corotation breakdown generates field aligned currents that are sufficient to give rise to auroral emissions is not constant around the planet and there exists no accurate relationship between the location of the $\mathrm{ME}$ and the corresponding source region in the magnetospheric plasma. Still, the shape of the contours, including the ME contour, gives important clues about the magnetic field. The latest inner magnetic field model, VIP4, was described by Connerney et al. [1998]. They suggested that the Jovian magnetic field may be represented by internal and external sources. Near the planet the magnetospheric field is dominated by the internal dynamo, while at greater distances the field is increasingly sensitive to external contributions, principally the field generated by the currents flowing in the magnetodisc and, to less extent, the field associated with the solar wind. The planetary internal field is represented by the VIP4 4th order spherical harmonic expansion. It was constrained by in situ magnetic field measurements from Voyager and Pioneer and remote information on the location of the infrared auroral footprint of Io. The contribution of the external field is estimated from an empirical model of the (magnetodisc) current sheet developed by Connerney et al. [1981], the solar wind field was neglected. These two main sources give rise to the "VIP4 plus Current Sheet model" described by Connerney et al. [1998]. For the sake of clarity, we will refer to it as the VIP4 model.

[5] VIP4 makes it possible to select an orbit in the equatorial plane and to project it in the ionosphere of Jupiter along the model field lines. It is thus possible to draw model reference contours corresponding to the orbits of Io, Europa and Ganymede and compare them with the observed auroral footpaths. Previous studies by Ballester et al. [1996], Clarke et al. [1998], and more recently Grodent et al. [2003] have shown a systematic deviation between the model and the observation of the northern aurora in a longitude sector ranging from System III (S3) longitudes $80^{\circ}$ to $150^{\circ}$ which the latter conveniently identify as the "kink sector". Grodent et al. [2003] further suggested that the distorted shape of the reference contours in this region may be attributed to the presence of a magnetic anomaly. However, the lack of observational constraints in this region prevented them from deriving the characteristics of such an anomaly. The new sets of HST images used in the present study makes it now possible to assess the presence of a magnetic anomaly and to derive some of its characteristics.

[6] The idea of a magnetic anomaly at the surface of Jupiter is not a recent one. Originally, i.e., before Voyager 1 encountered Jupiter, Dessler and Vasyliūnas [1979] predicted that several periodicities detected in charged particle measurements in the Jovian magnetosphere could be explained by invoking either a magnetic anomaly consisting of a weak field region in the northern high-latitude regions or a magnetodisc model. This idea was based on observations of clock-like phenomena in the Jovian magnetosphere, principally the detection of outflowing high-energy charged particles modulated with the Jovian rotation period. They suggested that the anomaly was centered at an S3 longitude of $\sim 230^{\circ}$, and extended over a longitude range (the active sector) of $120^{\circ}$. Post-voyager theoretical studies [Vasyliūnas and Dessler, 1981] confirmed that the magnetodisc model does not adequately explain the observations, and that a magnetic anomaly must be present. In more detail, we note that Hill and Dessler [1995] define the anomaly as "any feature of a planetary magnetic field that does not fit the simple dipole field pattern". According to this definition, the VIP4 model explicitly includes magnetic anomalies, since in addition to dipolar moment it comprises quadrupolar, octupolar and hexadecapolar moments. It has been proposed that the detection of a weak field magnetic anomaly be recognized by naming this the "Dessler anomaly" [Clarke et al., 2004].

[7] In the present study, we show auroral evidence of a magnetic anomaly characterized by a weak magnetic field near the northern auroral kink region and by a perturbation dipole.

\section{Observations and Data Reduction}

\subsection{Data Sets}

[8] In the present study we consider three HST data sets counting 1136 images of Jupiter's UV northern auroral region. They were all obtained with the photon-counting Multi-Anode Micro-channel Array of the High-Resolution Camera Solar Blind Channel (SBC) on the Advanced Camera for Surveys (ACS). The data were collected in the frame of several HST observation programs. The GO-10140 HST program was executed between April and May 2005; 77 images were taken during 5 HST orbits with the F125LP long-pass filter which is sensitive to the $\mathrm{H}_{2}$ Lyman and Werner bands but rejects emissions shortward of $125 \mathrm{~nm}$, therefore mostly excluding the $\mathrm{H}$ Lyman $-\alpha$ line. The GO-10507 HST program consisted of 77 F125LP images obtained between February and April 2006 and covering 4 HST orbits. The GO-10862 HST program is the largest continuous data set ever obtained for Jupiter's aurora. In this latter data set, we select 989 images of the northern hemisphere spanning 50 orbits and 5 months of observation starting in February 2007. This program mixes images captured with the F125LP and F115LP filters. The latter includes a contribution from the H Lyman- $\alpha$ line. 
[9] The average plate scale of 0.032 arcsec per pixel of ACS provides a field of view of $35 \times 31 \operatorname{arcsec}^{2}$ which is comparable to Jupiter's apparent equatorial diameter of $\sim 40$ arcsec. We usually require that one auroral region be placed near the field of view center in order to keep the global count rate from the sunlight reflected by the planetary disk below the limit. As a result, one quarter of Jupiter's planetary disk fits in the field of view, at maximum, and the planet center is never included in the images. Jupiter's internal magnetic field is strongly asymmetric and a better view of the auroral emissions is obtained from Earth orbit for the northern hemisphere than for the southern hemisphere. In addition, the auroral morphology of the southern hemisphere appears simpler than the northern hemisphere. The latter exhibits emission discontinuities and variations which may be attributed to the presence of a magnetic anomaly and which have no counterpart in the southern hemisphere. For these reasons, we concentrate on the images displaying the northern auroral region.

\subsection{Planet Center Finding}

[10] It is necessary to use a reliable reference frame fixed to the planet in order to compare the absolute location of auroral features on Jupiter. We choose to work with the common jovicentric coordinate system based on System III (S3) longitudes. The first step is accurately setting up this frame in individual images of Jupiter. This is usually performed by combining ephemeris information (using JPL NAIF ephemeris library) with HST attitude parameters stored in the headers accompanying each image file. However, the HST pointing accuracy is limited by the onboard guide star catalogue which can be up to $1 \mathrm{arcsec}$. This value is far too large for our purpose as it represents several tens of pixels while we are seeking a 1-pixel precision. Without any reference star in the FOV, the only way to precisely determine the location of the FOV central pixel is to use Jupiter's limb itself. Unfortunately, the FOV center of ACS was deliberately positioned high above the north pole of Jupiter in order to reduce the risks of damaging the detector with excessive count rates from the solar light reflected by the planetary disk. As a consequence, approximately three quarters of the planetary disk lies outside the image. The small remaining part includes the polar auroral region but not the central region of the disk. We use a fully automatic numerical method to estimate Jupiter's center position outside the field of view of ACS. This method was described by Bonfond [2007], it consists in detecting the limb on radial profiles and to fit a theoretical ellipse based on ephemeris to the limb points, taking the terminator into account. This multi-step method provides a non-subjective and systematic way of finding the center position in more than 1000 images with a 1 to 3-pixel accuracy level, comparable to that achieved with previous manual techniques.

\section{Reference Contours}

[11] Reference contours are important because they provide absolute magnetic mapping which makes it possible to link a latitude-longitude pair in the ionosphere to a precise region of the magnetosphere, near the equatorial plane. All the ACS images obtained during the three observation programs described in the previous section are analyzed individually. During this process, we manually select the central location of the footprints of Io, Europa and Ganymede, the median locus of Io's long trailing tail, and the median locus of the brightest continuous emission forming the main emission. The Europa footprint is significantly dimmer than that of Io and Ganymede (one order of magnitude, according to Grodent et al. [2006]) and its detection is often complicated or jeopardized by the presence of background auroral emission. In some cases we use the slower motion of Europa's footprint (Europa's orbital period is about 8 times larger than Jupiter's rotation period) to discriminate it from the rest of the quasi-corotating auroral emission. The location of these auroral features is then converted into jovicentric latitudes and S3 longitudes on Jupiter's tilted ellipsoid. We assumed an emission peak altitude of $700 \mathrm{~km}$ above the 1 bar pressure level for the satellite footprints and Io's tail, and $250 \mathrm{~km}$ for the ME. The $700 \mathrm{~km}$ altitude is deduced from images showing Io's footprint and/or its tail above the planetary sunlit limb [Bonfond et al., 2007b]. The $250 \mathrm{~km}$ altitude is compatible with the comprehensive study described by Grodent et al. [2001] combining multispectral images and modeling of the energy degradation of auroral electrons in Jupiter's polar atmosphere. This value was also determined from visible images of the aurora observed with the Galileo camera [Vasavada et al., 1999].

[12] The resulting jovicentric coordinates of each auroral feature are displayed on a polar map in Figure 1. They are fitted with three continuous reference contours drawn through the data points corresponding to Io, Europa and Ganymede (i.e., the auroral footprints of these satellites). The IFP and GFP contours are each based on $\sim 600$ data points, the EFP contour is defined with 144 points. The tail of Io is defined with 4805 points and the ME consists of 13170 data points.

[13] Table 1 lists the S3 longitudes and latitudes of the three satellites observed footprints as a function of the satellites S3 longitude. These values depict the reference contours appearing in Figure 1. For each satellite, the footprint positions are grouped in 10-degree bins centered on the satellite S3 longitude, and the footprint longitudes are averaged over each bin. In order to account for the uneven sampling of the data points, we derive a smooth satellitefootprint longitude trend characterizing the footprint location around the pole. The resulting longitude values are then assigned latitudes on the reference contour.

\subsection{Io Contour}

[14] To a large extent, the quasi-perfect alignment of the Io footprints and tail emission from the different images is expected. The magnetic shielding of Io in the inner magnetosphere, orbiting at $5.9 \mathrm{R}_{\mathrm{J}}$ outside the magnetodisc, implies that its overall morphology, materialized by its reference contour, is affected very little by external contributions such as the field produced by magnetospheric currents. It is clear though that intrinsic characteristics like the IFP brightness, the spots multiplicity and configuration [Gérard et al., 2006; Bonfond et al., 2007a, 2008] are changing with time and observing geometry, but, they do not affect the reference contour. In principle, the underlying complex mechanism could give rise to distribution of the multiple spots, owing to the different current paths, but this 


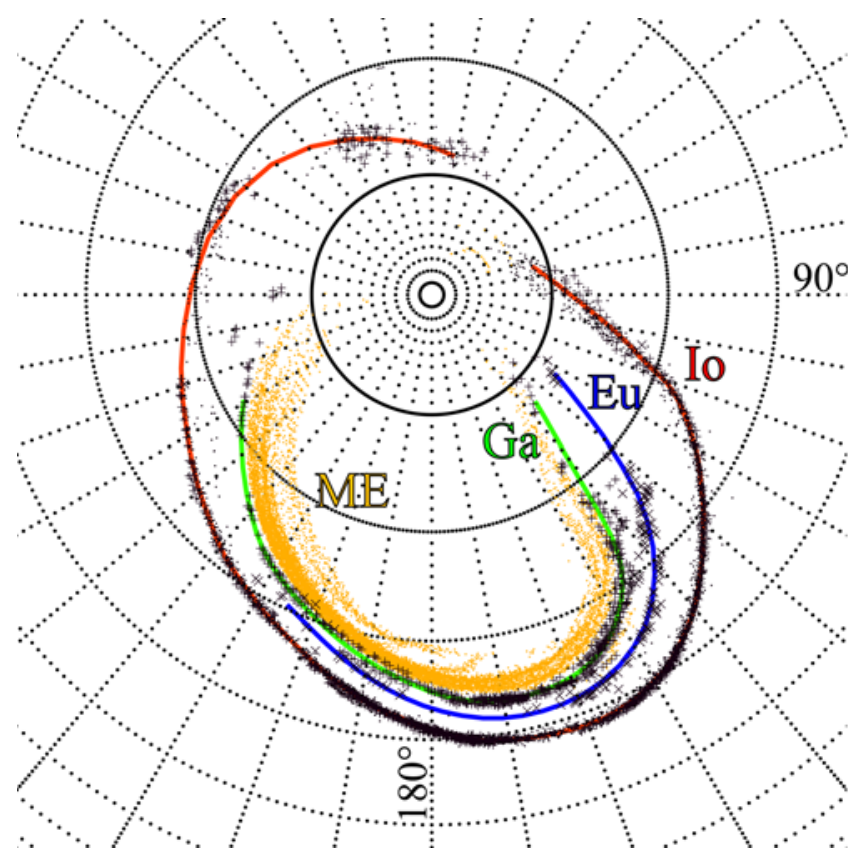

Figure 1. System III polar map of the UV auroral features obtained from the 1136 ACS images of Jupiter's northern polar region considered in the present study. A $10^{\circ}$-spaced grid is overplotted with $180^{\circ}$ System III toward the bottom and $90^{\circ}$ to the right. The footprint location of Io (red), Europa (blue), and Ganymede (green) is fitted with three continuous reference contours through their respective data point clouds. The location of Io's trailing tail is included to define Io's reference contour. Io footprint points are represented with black pluses, they form the equatorwardmost red contour and overlap Io's tail points marked with small dots. Europa foot points appear as black crosses, between Io's contour and Ganymede's contour. Ganymede (black plus) gives rise to the poleward-most green contour. The yellowish dots standing poleward of the Ganymede contour correspond to the main emission (ME).

S3 effect occurs mainly along the footpath, and the deviation perpendicular to the footpath is barely measurable with HST. The very low scatter of Io's footprint location across the contour, less than $2^{\circ}$, over such a large data set spanning 3 years of observation may then be considered as evidence for the high quality of the center finding method. This is a necessary prerequisite for the present analysis which supports our conclusions. Data points appearing at latitudes larger than $80^{\circ}$, between $\mathrm{S} 3$ longitudes $270^{\circ}-360^{\circ}$, and larger than $85^{\circ}$, near the $90^{\circ}$ meridian correspond to unfavorable viewing geometries where Io and/or its tail are very close to the planetary limb. For these configurations, the conversion from pixel position to planetocentric coordinates is highly inaccurate and the precision is on the order of $5^{\circ}$ in latitude and $10^{\circ}$ in longitude. However, these data points are extremely valuable because they correspond to previously unseen locations. They provide new information on the closure of Io's reference contour in a region which is barely visible from Earth orbit. The location of Io's trailing auroral tail was included to define Io's reference contour since it appears that the tail almost perfectly aligns with the footprint spot. Close inspection of the $70^{\circ}-120^{\circ}$ sector in Figure 1, and of the individual images displaying this region, consistently suggests that the contour has the shape of a kink, or more precisely it forms a hinge near latitude $78^{\circ}$ and the $110^{\circ} \mathrm{S} 3$ meridian. This inflection point breaks the contour and gives rise to the distorted shape described by Grodent et al. [2003]. This hinge will be interpreted later in the text as the signature of a localized perturbation of the surface magnetic field. The kink appears independently of the region near $0^{\circ}$, and thus of the $30-80^{\circ}$ gap. It is primarily the lack of symmetry with the region between $180^{\circ}$ and $270^{\circ}$, and the presence of a hinge of Io's footpath at $110^{\circ}$, confirmed by hundreds of data points from various observing geometries, which suggest the kink. The lack of symmetry is best seen in Figure 3a, where we compare the observed satellite footprints with the "symmetric" VIP4 footpaths.

\subsection{Europa Contour}

[15] So far, the location of Europa's footprint (EFP) has been seldom discussed, mostly because of the lack of detections. Grodent et al. [2006] provided reference positions for the $130^{\circ}-150^{\circ}$ System III sector, corresponding to

Table 1. System III Longitudes and Jovicentric Latitudes of the Observed Northern UV Footprints of Io, Europa, and Ganymede as a Function of the Satellites' System III Longitude; All Angles are in Degrees $^{\mathrm{a}}$

\begin{tabular}{|c|c|c|c|c|c|c|}
\hline \multicolumn{3}{|c|}{ Io $\left(5.9 \mathrm{R}_{\mathrm{J}}\right)$} & \multicolumn{2}{|c|}{ Europa $\left(9.4 \mathrm{R}_{\mathrm{J}}\right)$} & \multicolumn{2}{|c|}{ Ganymede $\left(15 \mathrm{R}_{\mathrm{J}}\right)$} \\
\hline $\begin{array}{c}\text { Satellite S3 } \\
\text { Longitude }\end{array}$ & $\begin{array}{c}\text { S3 } \\
\text { Longitude }\end{array}$ & Latitude & $\begin{array}{c}\text { S3 } \\
\text { Longitude }\end{array}$ & Latitude & $\begin{array}{c}\text { S3 } \\
\text { Longitude }\end{array}$ & \\
\hline 40.0 & 77.7 & 81.6 & & & 138.3 & 75.2 \\
\hline 50.0 & 95.9 & 76.6 & 123.7 & 77.1 & 138.9 & 74.4 \\
\hline 60.0 & 108.3 & 70.5 & 128.6 & 72.6 & 140.0 & 72.7 \\
\hline 70.0 & 118.0 & 64.9 & 133.1 & 66.7 & 141.7 & 69.0 \\
\hline 80.0 & 125.3 & 61.5 & 137.2 & 62.2 & 143.0 & 64.7 \\
\hline 90.0 & 131.1 & 58.7 & 140.5 & 59.5 & 144.6 & 61.9 \\
\hline 100.0 & 135.9 & 56.0 & 144.3 & 56.7 & 146.7 & 59.7 \\
\hline 110.0 & 141.2 & 53.2 & 148.2 & 54.7 & 149.2 & 58.0 \\
\hline 120.0 & 145.7 & 51.3 & 151.6 & 53.5 & 152.3 & 56.2 \\
\hline 130.0 & 149.9 & 49.9 & 155.1 & 52.5 & 155.5 & 54.9 \\
\hline 140.0 & 154.2 & 49.1 & 158.5 & 51.9 & 158.8 & 54.2 \\
\hline 150.0 & 158.7 & 48.6 & 161.7 & 51.6 & 162.2 & 53.6 \\
\hline 160.0 & 163.6 & 48.8 & 165.1 & 51.5 & 165.5 & 53.5 \\
\hline 170.0 & 168.5 & 49.1 & 168.8 & 51.5 & 169.0 & 53.5 \\
\hline 180.0 & 172.9 & 49.5 & 172.6 & 51.9 & 172.3 & 53.6 \\
\hline 190.0 & 177.9 & 50.5 & 176.5 & 52.5 & 175.6 & 54.0 \\
\hline 200.0 & 183.1 & 52.0 & 180.6 & 53.4 & 179.3 & 54.8 \\
\hline 210.0 & 188.9 & 53.3 & 184.6 & 54.5 & 183.2 & 55.8 \\
\hline 220.0 & 194.5 & 55.1 & 188.5 & 55.6 & 187.1 & 56.8 \\
\hline 230.0 & 200.0 & 56.7 & 192.8 & 57.0 & 191.1 & 57.8 \\
\hline 240.0 & 206.0 & 58.3 & 196.9 & 58.3 & 195.3 & 59.0 \\
\hline 250.0 & 212.5 & 60.0 & 201.6 & 59.6 & 199.8 & 60.2 \\
\hline 260.0 & 219.6 & 61.4 & 206.4 & 60.7 & 205.0 & 61.6 \\
\hline 270.0 & 227.9 & 63.5 & & & 210.5 & 62.9 \\
\hline 280.0 & 237.1 & 65.4 & & & 216.5 & 64.8 \\
\hline 290.0 & 247.0 & 67.0 & & & 222.5 & 66.8 \\
\hline 300.0 & 257.5 & 68.3 & & & 229.0 & 68.7 \\
\hline 310.0 & 268.5 & 69.5 & & & 235.9 & 70.6 \\
\hline 320.0 & 280.3 & 70.2 & & & 243.0 & 72.4 \\
\hline 330.0 & 292.9 & 71.0 & & & 250.6 & 74.9 \\
\hline 340.0 & 306.9 & 72.3 & & & & \\
\hline 350.0 & 322.2 & 74.1 & & & & \\
\hline
\end{tabular}

${ }^{a}$ These footprint positions correspond to the three reference contours drawn in Figures 1, 3, and 4. They are values averaged over 10-degree longitude bins (see text). 
the low-latitude part of the kink region. They showed that in this sector, the footpath of Europa is poleward, since Europa is orbiting at $\sim 9.4 R_{J}$, and almost parallel to that of Io. The present data set considerably extends the sector where the EFP is detected to $120^{\circ}-210^{\circ}$ (Figure 1). It should be noted that even when the viewing geometry is favorable for Europa, it is not always possible to detect its auroral footprint. It is difficult to establish strong statistics of the EFP location because of the very low-footprint brightness, around $10 \mathrm{kR}$ above the background level, and of the presence of bright and variable diffuse auroral emissions, mostly in the kink region, and the occurrence of isolated auroral spots which can be easily mistaken for a footprint. Many of the new data points collected in the large 2007 data set correspond to a region around the $180^{\circ}$ meridian where the lowest latitudes are reached. The remaining points confirm, and somewhat refine the contour in the kink region presented by Grodent et al. [2006]. To first order, in this extended range of longitudes, the Europa footpath remains almost parallel to Io's reference contour. However, closer inspection of the contours reveals a departure in the kink region. While the contour of Io forms a broken shape near the $110^{\circ}$ hinge discussed above, the footpath of Europa converges to a straight line toward the jovigraphic pole. We point out that the straight line trend in the $125^{\circ}-130^{\circ}$ footpath sector is a first-order approximation. The presence of isolated data points a couple of degrees poleward of the contour may suggest that the actual contour is slightly curved poleward. As will be discussed below, this localized divergence of the contours is important for the modeling of the surface magnetic field since it suggests the presence of a localized magnetic perturbation.

\subsection{Ganymede Contour}

[16] The reference contour of Ganymede's footprint (GFP) is particularly important because Ganymede's orbital radius is about $15 R_{J}$, and its footprint always appears very close to the main emission, but always equatorward of it. It thus provides one with a convenient landmark for locating the sub-corotating magnetospheric plasma giving rise to the $\mathrm{ME}$ through the corotation enforcement mechanism. In addition, diffuse auroral emissions are often located near the GFP reference contour. A secondary emission appearing equatorward of the ME, referred to as the secondary oval [Grodent et al., 2003; Tomas et al., 2004] also shows up near the GFP footpath. Since Ganymede is the farthest Galilean moon having a measurable auroral footprint, its footprint is often used as a proxy to adjust the magnetic local time (MLT) in the vicinity of Ganymede's orbit [e.g., Grodent et al., 2004] or at least to roughly take the bending of the field lines off the meridian plane into account in the middle magnetosphere. Figure 1 displays the GFP contour poleward of the EFP. The available data points span a region from approximately $130^{\circ}$ to $270^{\circ}$ longitude. However, as for the footprint of Io, the data points lying at a latitude larger than $75^{\circ}$ were obtained when the footprint was very close to the planetary limb and are therefore affected by an uncertainty of $\pm 2^{\circ}$ in latitude and $10^{\circ}$ in longitude. The reference contour of Ganymede is almost parallel to that of Io and Europa, particularly beyond $145^{\circ}$. The observed behavior is different in the kink region where the contour tends to follow a straight line toward the pole, similar to the case of
Europa (with the same possible slight curvature poleward), and unlike Io. Accordingly, the GFP reinforces the divergent trend of the contours in this perturbed sector.

[17] The reference contours of Ganymede, and to some extent of Europa, are average curves passing through scattered data points. In the case of Ganymede the scatter is on the order of $2^{\circ}$ across the contour near $170^{\circ}$. This value is in agreement with the changes observed by Grodent et al. [2008] who suggested that observed variations of the current density in the current sheet by a factor of 3 and/or of the current sheet thickness are likely to perturb the magnetic field topology and give rise to a latitudinal shift of the GFP on order of $2^{\circ}$. Similarly, such variations of the current sheet will produce a smaller shift $\sim 1.5^{\circ}$ of the EFP and a barely measurable IFP shift less than $1^{\circ}$. The changes in the current sheet simultaneously shift the footprint of Ganymede (Europa and Io) and the location of the ME. Therefore it is very unlikely that the magnetic field topology can be deformed in such a manner as to put the GFP poleward of the ME.

\section{The Main Auroral Emission}

[18] There are at least three reasons for not deriving a unique reference contour for the ME. First, contrarily to the satellite footprints, it has not been demonstrated that the $\mathrm{ME}$ maps to a constant distance in the magnetosphere. The fact that it always appears poleward of the footprint of Ganymede simply indicates that it maps to sub-corotating magnetospheric plasma beyond the orbit of Ganymede. Galileo observations of the plasma flow in the equatorial plane [Krupp et al., 2001] strongly suggest that the distance at which corotation significantly breaks down depends on local time. If one assumes that the ME location is directly linked to this radial distance, then it is expected that the $\mathrm{ME}$ maps to distances varying from $20 \mathrm{R}_{\mathrm{J}}$ near noon [Nichols and Cowley, 2004] to several tens of $\mathrm{R}_{\mathrm{J}}$ in the nightside sector [Fukawaza et al., 2005]. Additionally, HST observations have shown that the ME brightness changes with local time as a result of the field aligned currents distribution [Radioti et al., 2008]. Second, the influence of the internal magnetic field quickly decreases with radial distance. Beyond the orbit of Ganymede the total magnetic field is strongly influenced by external contributions, mainly from the current sheet and magnetopause currents. The GFP latitudinal shift induced by variations of the current sheet is stronger for the ME. Grodent et al. [2008] showed that an emission mapping to $25 \mathrm{R}_{\mathrm{J}}$ is shifted poleward by about $3^{\circ}$ at $170^{\circ}$ of longitude when the current density of the current sheet is divided by 3 (compared to the value considered in VIP4). Other mechanisms such as the confinement of the equatorial plasma by the magnetopause are sensitive to the solar wind activity. They also influence the latitudinal extent of the ME [Grodent et al., 2003] through the plasma flow distribution and, altogether, might explain the scattering of data points in Figure 1. The third reason stems from the varying morphology of the ME in the kink region. Figure 1 shows the data points that were selected along the brightest emissions forming the ME. The smaller concentration of data points at longitudes below $160^{\circ}$ does not result solely from a biased sampling of the CML near $180^{\circ}$, but also from an intrinsic temporal variation such that the ME does not always exist in the kink region or at least does not 


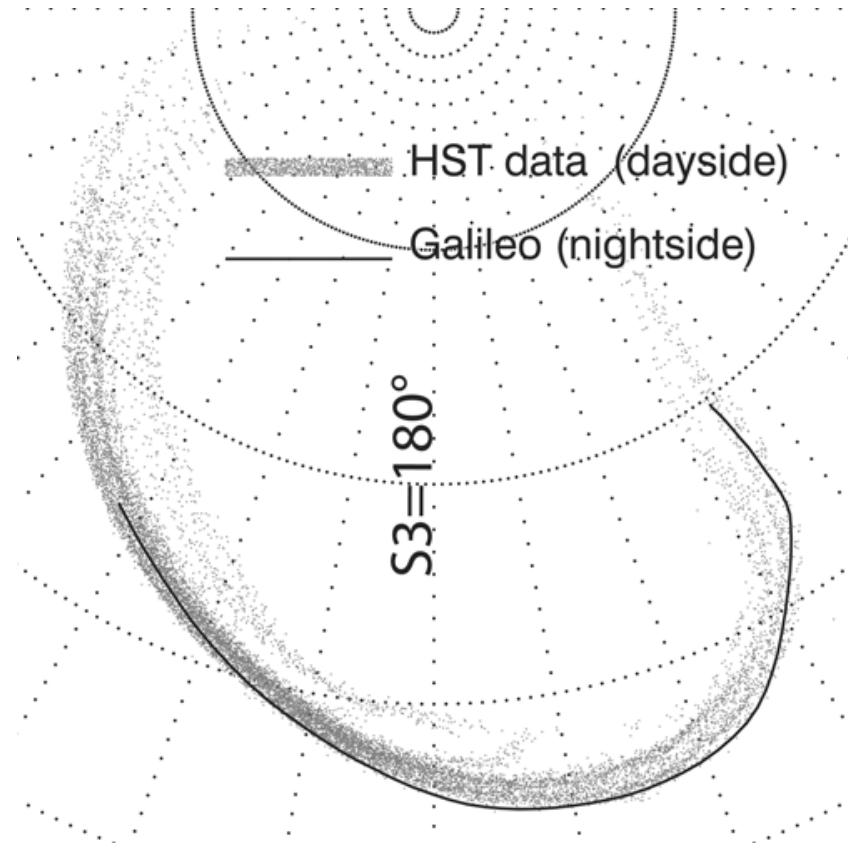

Figure 2. System III polar map of the main emission data points obtained from the HST UV images (grey dots) in the dayside. The solid line represents the location of the main emission observed in the nightside with the Galileo spacecraft in the visible wavelength range. The superposition of the dayside HST ME and the nightside Galileo $\mathrm{ME}$ on this S3 map demonstrates that the broken appearance of the auroral emission in the kink sector prevails at all CMLs. Therefore it is not a local time effect but a surface magnetic field effect. A $10^{\circ}$-spaced grid is overplotted with $180^{\circ} \mathrm{S} 3$ toward the bottom and $90^{\circ}$ to the right.

always form a continuous narrow arc of emission. A striking example is given by Grodent et al. [2008] (Figure 1) where 2 images obtained with quasi-identical viewing geometries, but separated by more than 4 years, display dissimilar morphologies in the kink sector. Having given arguments against a ME contour, it is nonetheless worth comparing the ME overall morphology with the satellite contours. This comparison is straightforward since, as already mentioned, the ME points are always poleward of the contour of Ganymede and follow the same straight line trend in the kink region between $140^{\circ}$ and $150^{\circ}$ of longitude. However, it should be noted that in this latter sector, individual polar maps often show that the $\mathrm{ME}$ is broken down and that the straight line is a first approximation. The morphologies significantly diverge at longitudes larger than $230^{\circ}$ where the ME tends to turn toward the pole more rapidly than the GFP contour. This may be interpreted as an effect of the increasing corotation breakdown distance in the nightside for this longitude sector which is usually observed when it maps to the predawn sector [Krupp et al., 2001]. It should be remembered, however, that this region is imaged near the planetary limb where the location inaccuracy rapidly grows.

[19] In-situ observations of the main auroral emission in the visible wavelength range were obtained in the night side hemisphere of Jupiter with the Galileo spacecraft in
November 1997 [Vasavada et al., 1999]. During Galileo's orbit E11, the kink sector was imaged when it appeared before dawn. In this configuration, the main auroral emission was located near the limb and one should consider a mapping precision of a couple of degrees owing to the presence of guide stars in Galileo's field of view. Figure 2 compares the ME location deduced from Galileo orbit E11 with the HST ME data points. The overlap is substantial and confirms that the distorted shape of the auroral emission in the kink sector is preserved on the nightside. The immediate and important consequence of this result is that the distortion "rotates" with the planet, that is, it is primarily imposed by the surface magnetic field. This feature advocates for the presence of a local magnetic disturbance in the kink sector and is addressed in the discussion section."

\section{Discussion}

\subsection{Local Time Effects}

[20] At this point of the discussion, we should insist that we do not rule out the possibility that LT effects significantly alter the auroral morphology, especially the ME. Grodent et al. [2003] and Radioti et al. [2008] suggested that the observed asymmetries in the magnetospheric plasma flows may be responsible for the fine structure of the $\mathrm{ME}$, with a narrow continuous arc of emission in the dawn sector contrasting with broader, patchy emissions in the dusk sector and a region devoid of emission mapping to 813LT. Therefore the reality is most likely a complex combination of LT and S3 behaviors. The S3 dependence controls the general shape of the footpath and gives rise to a "rigid S3 auroral frame". The LT behavior on its side has some effect on the brightness distribution along this S3 frame. The present study emphasizes the S3 facet and the fact that the kink structure is a S3 effect rather than LT. It is clear that magnetospheric dynamics influences the auroral morphology as well. Substorm-like events [Grodent et al., 2004] and injections [Mauk et al., 2002] have been shown to be associated to transient auroral features. However, a steady state feature like the kink, fixed in S3, could hardly be related to dynamic effects occurring at a specific LT region (substorm-like events) [Woch et al., 2002] or to phenomena randomly distributed in S3 longitudes (particle injections) [Mauk et al., 1999].

[21] As far as the satellite footprints are concerned, it is important to remember that the mechanisms and the magnetospheric plasma population involved in the production of auroral footprints are most likely different from those giving rise to the ME. They are much less influenced by the asymmetries in the plasma flows and densities that have been observed by Galileo beyond the orbit of Ganymede [Krupp et al., 2001].

[22] In order to reproduce the observed deviation of Io's footpath in the kink region for a restricted range of CMLs $\left(80^{\circ}-240^{\circ}\right)$, one would have to assume that this LT process is confined to a specific region of the magnetosphere. However, at least two arguments are challenging this hypothesis. First, it should also produce some measurable deviations of the footpath(s) in the southern hemisphere. However, so far this has never been reported. Second, every time a satellite crosses this region, the location of its footprint should be perturbed. However, the observed foot- 

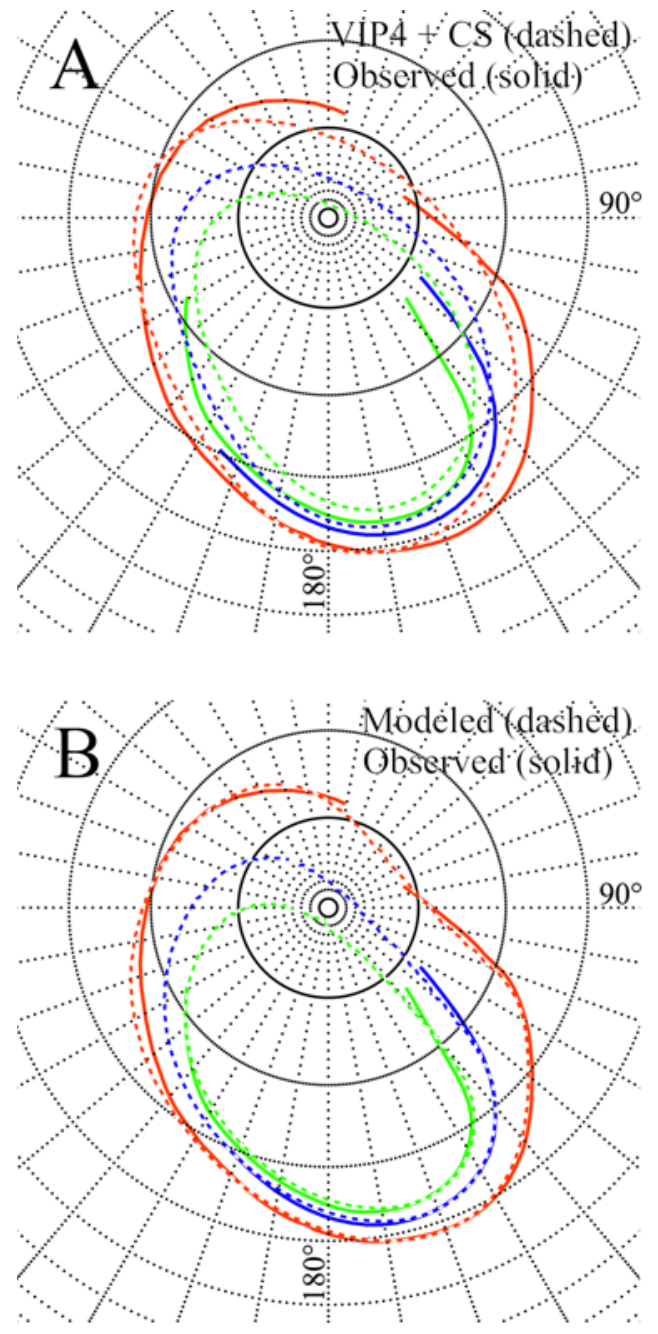

Figure 3. System III polar map comparing the observed UV contours of Io (solid red), Europa (solid blue), and Ganymede (solid green) with (a) the corresponding footpaths derived from the VIP4 + current sheet (CS) magnetic field model (dashed red, blue, green closed contours), and with (b) the perturbed magnetic field model (dashed red, blue, green closed contours). The VIP4 + CS model footpaths roughly agree with the observed contours. However, a closer inspection reveals substantial inconsistencies in the kink sector between S3 longitudes $80^{\circ}$ and $150^{\circ}$ (bottom right quadrant), and more generally for the Europa and Ganymede footprints. The agreement is considerably improved with the perturbed field model, especially in the kink sector where the combination of the reduced field and the localized perturbation (the magnetic anomaly) makes it possible to reproduce the divergence of the observed contours. The Ganymede and Europa footprints are reasonably accounted for by the perturbed model field. A $10^{\circ}$-spaced grid is overplotted with $180^{\circ} \mathrm{S} 3$ toward the bottom and $90^{\circ}$ to the right.

prints of Io, and to some extent of Ganymede, in a given range of S3 longitude when Io is in this sector perfectly overlap the footprints which are observed for the same range of S3 longitude but when Io is outside this sector. This overlap is observed all along the footpath, i.e., at all S3 longitudes, and strongly suggests that while LT effects could increase the scattering of the data points and thus increase the width of the footpaths, the distorted shape of the footpaths in the kink region drawn in Figure 1 cannot be caused by LT effects.

\subsection{Evidence for a Magnetic Anomaly}

[23] Figure 1 shows that the satellite reference contours are almost parallel but in the kink sector, between S3 $80^{\circ}$ and $150^{\circ}$, they tend to diverge. The main auroral emission follows the same general trend as the satellite footprints. Its morphology is highly variable in the kink sector, ranging from a bright narrow arc to a diffuse patchy and weak emission, passing through intermediate distributions at different locations. Grodent et al. [2003] showed that the average ME brightness is usually larger at $\mathrm{S} 3$ longitudes $<180^{\circ}$ (the kink sector) than at longitudes $>180^{\circ}$. A brighter, more variable and less structured auroral emission is compatible with a weaker surface magnetic field since the resulting lower altitude of the magnetic mirror point allows more auroral electrons to reach the ionosphere.

[24] We tested the hypothesis of a surface magnetic field weaker than the field calculated from the VIP4 model [Connerney et al., 1998]. The VIP4 (plus current sheet) model allows us to calculate reasonable footprint positions and has been widely used to map the auroral emissions down to the equatorial plasma. However, VIP4 is known to become increasingly inaccurate beyond the orbit of Io, mainly because the current sheet contribution is based on a symmetric and static magnetodisc field. For Europa and Ganymede, one expects a larger role for the external field than for Io. Outside the middle magnetosphere, beyond $\sim 25-30 \mathrm{R}_{\mathrm{J}}$, local time-dependent external field contributions become dominant and asymmetries in the field in local time, due to the solar wind interaction with Jupiter's magnetic field, are expected to further distort the field lines. In that regard, Grodent et al. [2008] suggested that variations of the current density in the current sheet and/or of the current sheet thickness, within the limits of in situ observations, are likely to perturb the magnetic field topology. If the current sheet is decreased by a factor of three, or if its thickness is halved (or a combination of both), then it may give rise to a poleward shift of about $1^{\circ}$ for the EFP, $2^{\circ}$ for the GFP, and on order of $3^{\circ}$ for the ME.

[25] In the northern kink region, from $0^{\circ}$ to $150^{\circ}$, VIP4 was constrained by only two IFP data points. Therefore it is not surprising that in this region the calculated satellite footpaths do not adequately match the UV observations presented in this study. Figure 3a shows that the major deviation between the observed and VIP4 footpaths of Io, Europa and Ganymede, is a significant poleward shift of the UV emissions that diverges from the smooth oval-like shape of the VIP4 footpaths.

[26] We manually adjusted the spherical harmonic coefficients in order to improve the overlap between model and observations in the kink region of the northern hemisphere of Jupiter. The main changes were made on the octupolar $(1=3)$ and hexadecapolar $(1=4)$ terms, some of the lower order coefficients were also modified but the difference is within the range of published values. The poleward shift is relatively well reproduced if the coefficients are modified in such a manner as to decrease the surface field in the northern kink region. However, this is not sufficient because 
Table 2. Characteristics of Two Possible Perturbation Dipoles in Spherical Coordinates $(r, \theta, \phi)$, Following the Formalism Given by Dyment and Arkani-Hamed [1998] ${ }^{\mathrm{a}}$

\begin{tabular}{lc}
\hline \multicolumn{1}{c}{$\begin{array}{c}\text { Dipole Source } \\
\text { Point Position }\end{array}$} & $\begin{array}{c}\text { Magnetic Moment } \\
\text { Components (Gauss) }\end{array}$ \\
\hline$r=0.755 \mathrm{R}_{\mathrm{J}}\left(\right.$ depth $\left.=0.245 \mathrm{R}_{\mathrm{J}}\right)$ & $M_{r}=0.01$ \\
$\theta=26^{\circ}($ colatitude) & $M_{\theta}=0.03$ \\
$\phi=107^{\circ}(\mathrm{S} 3)$ & $M_{\phi}=0.037$ \\
$r=0.755 \mathrm{R}_{\mathrm{J}}\left(\right.$ depth $\left.=0.245 \mathrm{R}_{\mathrm{J}}\right)$ & $M_{r}=-0.02$ \\
$\theta=22^{\circ}($ colatitude) & $M_{\theta}=0.015$ \\
$\phi=120^{\circ}(\mathrm{S} 3)$ & $M_{\phi}=0.045$ \\
\hline
\end{tabular}

${ }^{a}$ The upper rows correspond to the dipole equatorward of the Io footpath discussed in detail in the present study. The lower rows characterize an alternative dipole, poleward of the Io contour, in better agreement with a probable poleward curvature of the EFP and GFP footpaths in the anomaly sector. The total magnitude is $\sim 0.05 \mathrm{GR}_{\mathrm{J}}{ }^{3}$ in both cases.

any change in the coefficients, at least these for $1 \leq 4$, will simultaneously alter the footpaths of Io, Europa and Ganymede. In other words by changing the $1 \leq 4$ coefficients we cannot reproduce the divergence of the footpaths, nor the IFP hinge. In addition, previous studies of the southern auroral emissions [Clarke et al., 1998; Grodent et al., 2003] suggest that there is no such divergence of the southern footpaths at magnetically conjugate locations. Accordingly, it is necessary to consider a change in the model magnetic field which does not significantly affect the southern surface field.

[27] The simplest way to account for the distorted and diverging northern footpaths consists in decreasing the surface magnetic field as described above and adding a small localized magnetic perturbation to the internal magnetic field. We consider the confined influence of a small dipole rigidly rotating with the planet just below the surface. The expression of the magnetic anomaly caused by this dipole follows the formulation of Dyment and ArkaniHamed [1998] which allowed us to adjust the dipole source position in Jupiter's coordinates, as well as the magnitude and orientation of its magnetic moment. These parameters are listed in Table 2. They were specifically set in order to restrict its influence to the kink region and to reproduce the Io footprint hinge near $78^{\circ}$ of latitude and $110^{\circ}$ of S3 longitude without affecting Europa and Ganymede's footpaths. We consider a dipole source near the hinge, actually equatorward of the footpath of Io at a latitude of $64^{\circ}$ and a longitude of $107^{\circ}$. Its main effect is to drag the field lines passing by the hinge toward the equator. Its region of influence is controlled by the depth of the dipole; the deeper it is, the larger its range, provided its moment is increased accordingly. A reasonable compromise is reached by setting the depth to $0.245 \mathrm{R}_{\mathrm{J}}$ below the surface and the magnitude to $\sim 0.05 \mathrm{GR}_{\mathrm{J}}^{3}$, i.e., $\sim 1 \%$ of Jupiter's dipole moment. Its projection on the surface is approximately oriented along the normal to Io's footpath at the level of the hinge and has a small radial component off the planet. It thus locally increases the unperturbed surface magnetic field. Figure $4 \mathrm{~b}$ shows a polar projection of the perturbed northern surface magnetic field magnitude and the new calculated reference contour of Io (to be compared with the unperturbed VIP4 model in Figure $4 \mathrm{a})$. The magnetic anomaly created by the dipolar perturbation appears as an isolated outgrowth near the $100^{\circ}$ meridian. Its magnitude is relatively large compared to the unperturbed field and reaches $\sim 70 \%$ of the maximum value. However, its influence rapidly falls away from the


Figure 4. System III polar map of the surface magnetic field intensity (Gauss) obtained with the VIP4 plus current sheet model (a) [Connerney et al., 1998] and with the perturbed model deduced from the present study (b). The VIP4 footpath of Io is overplotted as a solid line in Figure 4a and the observed footpath of Io deduced from HST images is drawn with black dots in Figure 4b. The major differences are the lessening of the surface field about the $120^{\circ} \mathrm{S} 3$ meridian between $60^{\circ}$ and $90^{\circ}$ latitude, the increased and slightly displaced maximum field near $160^{\circ}$, and the presence of a localized perturbation dipole field equatorward of the footpath of Io at a latitude of $64^{\circ}$ and a longitude of $107^{\circ}$. A $10^{\circ}$-spaced grid is overplotted with $180^{\circ} \mathrm{S} 3$ toward the bottom and $90^{\circ}$ to the right. The same color table applies to both figures. 
anomaly so that only the footpath of Io is effectively deviated, consistent with the UV observations. We note that the depth of the perturbation dipole $\left(0.755 \mathrm{R}_{\mathrm{J}}\right.$ from the center) is compatible with the outer limit of the dynamo region which is currently estimated to lie at $0.85 \mathrm{R}_{\mathrm{J}}$ [Guillot et al., 2005]. The presence of such dipole, inferred from auroral observations, could constitute a useful constraint on the location of the dynamo layer.

[28] Figure $3 \mathrm{~b}$ displays the observed reference contours of Io, Europa and Ganymede and compares them with the footpaths calculated with the perturbed field model. The agreement is particularly good in the kink, or anomaly region, as expected. We did not attempt to perfectly fit the contours (i.e., to less than $2^{\circ}$ difference) beyond $220^{\circ}$ since it would involve complex generalized inversion techniques to adjust the high-order coefficients of the spherical expansion, which is beyond the scope of the present study (this will be the subject of future analysis including the southern hemisphere). We note however that our rough adjustment of the spherical harmonic coefficients in the anomaly region gives rise to a footpath of Io which is compatible with the footprints observed near $300^{\circ}$. It also provides a reasonable solution for closing the three contours in the regions devoid of observational constraints.

[29] It is clear that the above solution is not unique. Other configurations of the perturbing dipole and of the internal field may match the observations as well. We derive another configuration, with the same spherical harmonic development, which is consistent with possible poleward curvature of the EFP and GFP in the anomaly sector, rather than straight lines. The parameters of this dipole are also listed in Table 2. The major difference stems from the location of the dipole source, which, contrarily to the previous case, is slightly poleward of the Io contour, at S3 longitude $120^{\circ}$ and latitude $68^{\circ}$. We also note that, while the total magnitude remains almost unchanged, the radial component is reversed and there is a transfer of magnetic moment from the azimuthal to the latitudinal component. Overall, these two solutions are comparable. They show that even if we are unable to derive the precise description of the perturbation dipole, it is necessary to consider that such perturbation exists in the anomaly region.

[30] The lessening of the VIP4 surface magnetic field in the anomaly/kink region, the increased maximum field near $160^{\circ}$, and the addition of a localized dipolar perturbation near the surface thus represent a plausible approach to account for the distorted shape of the observed reference contours in the northern hemisphere. In the present method, we only concentrated on the auroral morphology and we do not take into account in situ constraints on the magnetic field near the equatorial plane. Nevertheless, the changes that we brought to the surface magnetic field have modest impact on the equatorial plane field. Inside the orbit of Ganymede, from 6 to $15 \mathrm{R}_{\mathrm{J}}$, the difference is less than $10 \%$ which is overall less than the magnetic effects of the current sheet intrinsic variability. The perturbed equatorial field is larger than the VIP4 field, mainly as an effect of the $1.6 \%$ increase of the first-order spherical harmonic moment. The modifications that are applied to the VIP4 dipolar coefficients propagate in the equatorial plane at all distances. The quadrupolar and higher order terms have almost no effect on the ambient field and the perturbation dipolar field contrib- utes about $1 \%$, which is almost 5 times less than the effect of the changes made on the VIP4 dipolar coefficients. Overall, these changes of the equatorial magnetic field are small compared to the accuracy of in situ measurements resulting from instrumental limitations and the intrinsic temporal and spatial variability of the total field.

\section{Summary}

[31] We provide a first-order model of a new magnetic anomaly in the northern hemisphere of Jupiter. The presence of such anomaly is inferred from the detailed analysis of more than 1000 UV HST images of the auroral emissions appearing in the northern hemisphere. The data points corresponding to the footprints of Io, Europa and Ganymede, observed under different viewing geometries, form individual auroral footpaths which are fitted with three reference contours. The auroral footpath of Io forms a kink in a region bounded by S3 longitudes $80^{\circ}$ and $150^{\circ}$ which we identify as the kink or anomaly region. We find that the footpath exhibits a hinge near latitude $78^{\circ}$ and the $110^{\circ} \mathrm{S} 3$ meridian. This inflection point breaks the contour and gives rise to the distorted shape of the reference contour. The reference contours of Europa and Ganymede are inferred from a much larger range of longitudes than in previous studies. They are shown to be almost parallel to that of Io, particularly beyond $145^{\circ}$. However, they diverge in the kink region: while the IFP forms a kink away from the pole, the polar projected GFP and EFP paths tend to follow straight lines toward the pole. We do not define a reference contour for the ME, because unlike the satellite footprints, it is most probable that this emission does not map to a constant distance. In addition, intrinsic temporal variations of the emission are such that the ME does not always exist in the kink region or at least does not always form a continuous narrow arc of emission. The ME points are always poleward of the contour of Ganymede and follow the same straight line trend in the kink region between $140^{\circ}$ and $150^{\circ}$ of longitude.

[32] The simplest way to account for the distorted and diverging northern footpaths consists in decreasing the VIP4 surface magnetic field in the kink/anomaly region and adding a localized magnetic perturbation or anomaly below the surface. A good fit of the modeled to the observed footpaths is obtained by using a magnetic dipole source positioned at $64^{\circ}$ latitude and $107^{\circ}$ longitude, or alternatively at $68^{\circ}$ latitude and $120^{\circ}$ longitude. This anomaly rotates with the planet (i.e., it is fixed in S3) at a depth of $0.245 \mathrm{R}_{\mathrm{J}}$ below the 1-bar level. Its magnitude is estimated to $\sim 0.05 \mathrm{GR}_{\mathrm{J}}^{3}$, which is $\sim 1 \%$ of Jupiter's dipole moment. The decreased surface field in the kink region implies a lower altitude of the mirror point near the ionosphere, and an augmented influence of the fluctuating contribution of the current sheet to the total magnetic field near the equatorial plane. This is consistent with the observed brighter, more variable and less structured main auroral emissions (mapping to beyond the orbit of Ganymede) in the kink/anomaly region. Finally, we note that the changes brought to the VIP4 plus current sheet model have a small influence on the magnetic field in the equatorial plane, in comparison with the accuracy of in situ measurements resulting from instrumental limitations and the intrinsic temporal and spatial 
variability of the total field. As a result, they do not challenge previous in situ observations.

[33] Apart from a series of 8 images obtained by the Galileo spacecraft, the present study is limited to the dayside auroral emissions by the vantage point of HST near Earth orbit. Crucial and accurate information about the nightside emissions and their origin can only be obtained by in situ observatories following high-latitude trajectories. This viewing geometry will be reached by NASA's New Frontiers JUNO spacecraft. Its polar orbit will enable complete mapping of the asymmetries and detailed structure of Jupiter's magnetic field and will make it possible to measure the precipitating charged particles into the atmosphere that generate the complex auroral patterns.

[34] Acknowledgments. This work is based on observations with the NASA/ESA Hubble Space Telescope, obtained at the Space Telescope Science Institute (STScI), which is operated by AURA, Inc., for NASA under contract NAS5-26555. DG, BB, JCG, AR, and JG are supported by the Belgian Fund for Scientific Research (FNRS) and by the PRODEX Program managed by the European Space Agency in collaboration with the Belgian Federal Science Policy Office. Work in Boston was supported by grant HST-GO-10862.01-A from the Space Telescope Science Institute to Boston University. Wolfgang Baumjohann thanks Jamesina Simpson and another reviewer for their assistance in evaluating this paper.

[35] Wolfgang Baumjohann thanks Norbert Krupp and W. R. Pryor for their assistance in evaluating this paper.

\section{References}

Ballester, G. E., et al. (1996), Time-resolved observations of Jupiter's farultraviolet aurora, Science, 274, 409-412.

Bonfond, B. (2007), The Io FUV footprint: Analysis of Hubble Space Telescope observations of Jupiter's aurorae, M.S. thesis, 92 pp., Université de Liège, Liège, Belgium.

Bonfond, B., J.-C. Gérard, D. Grodent, and J. Saur (2007a), Ultraviolet Io footprint short timescale dynamics, Geophys. Res. Lett., 34, L06201, doi:10.1029/2006GL028765.

Bonfond, B., J.-C. Gérard, and D. Grodent (2007b), Morphology of the Io footprint, European Geosciences Union General Assembly 2007, Vienna, Austria, 15-20 April 2007.

Bonfond, B., D. Grodent, J.-C. Gérard, A. Radioti, J. Saur, and S. Jacobsen (2008), UV Io footprint leading spot: A key feature for understanding the UV Io footprint multiplicity?, Geophys. Res. Lett., 35, L05107, doi:10.1029/2007GL032418.

Clarke, J. T., et al. (1998), Hubble Space Telescope imaging of Jupiter's UV aurora during the Galileo orbiter mission, J. Geophys. Res., 103, 20,217-20,236.

Clarke, J. T., et al. (2002), Ultraviolet auroral emissions from the magnetic footprints of Io, Ganymede, and Europa on Jupiter, Nature, 415, 997-1000.

Clarke, J. T., D. Grodent, S. Cowley, E. Bunce, P. Zarka, J. Connerney, and T. Satoh (2004), Jupiter's aurora, in Jupiter: The Planet, Satellites and Magnetosphere, edited by F. Bagenal, B. McKinnon, and T. Dowling, pp. 639-670, Cambridge Univ. Press, New York.

Connerney, J. E. P., M. H. Acuña, and N. F. Ness (1981), Modeling the Jovian current sheet and inner magnetosphere, J. Geophys. Res., 86, $8370-8384$.

Connerney, J. E. P., M. H. Acuña, N. F. Ness, and T. Satoh (1998), New models of Jupiter's magnetic field constrained by the Io flux tube footprint, J. Geophys. Res., 103, 11,929-11,939.

Cowley, S. W. H., and E. J. Bunce (2001), Origin of the main auroral oval in Jupiter's coupled magnetosphere-ionosphere system, Planet. Space Sci., 49, 1067-1088.

Dessler, A. J., and V. M. Vasyliūnas (1979), The magnetic anomaly model of the Jovian magnetosphere: predictions for Voyager, Geophys. Res. Lett., 6, 37-40.

Dyment, J., and J. Arkani-Hamed (1998), Equivalent source magnetic dipoles revisited, Geophys. Res. Lett., 25(11), 2003-2006.

Fukazawa, K., T. Ogino, and R. J. Walker (2005), Dynamics of the Jovian magnetosphere for northward interplanetary magnetic field (IMF), Geophys. Res. Lett., 32, L03202, doi:10.1029/2004GL021392.
Gérard, J.-C., V. Dols, F. Paresce, and R. Prangé (1993), Morphology and time variation of the Jovian far UV aurora: Hubble Space Telescope observations, J. Geophys. Res., 98(E10), 18,793-18,801.

Gérard, J.-C., A. Saglam, D. Grodent, and J. T. Clarke (2006), The morphology of the ultraviolet Io footprint emission and its control by Io's location, J. Geophys. Res., 111, A04202, doi:10.1029/2005JA011327.

Grodent, D., J. H. Waite Jr., and J.-C. Gérard (2001), A self-consistent model of the Jovian auroral thermal structure, J. Geophys. Res., 106, $12,933-12,952$.

Grodent, D., J. T. Clarke, J. H. Waite, J. Kim, and S. W. H. Cowley (2003), Jupiter's main auroral oval observed with HST-STIS, J. Geophys. Res., 108(A11), 1389, doi:10.1029/2003JA009921.

Grodent, D., J.-C. Gérard, J. T. Clarke, G. R. Gladstone, and J. H. Waite Jr. (2004), A possible auroral signature of a magnetotail reconnection process on Jupiter, J. Geophys. Res., 109, A05201, doi:10.1029/2003JA010341.

Grodent, D., J.-C. Gérard, J. Gustin, B. H. Mauk, J. E. P. Connerney, and J. T. Clarke (2006), Europa's FUV auroral tail on Jupiter, Geophys. Res. Lett., 33, L06201, doi:10.1029/2005GL025487.

Grodent, D., J.-C. Gérard, A. Radioti, B. Bonfond, and A. Saglam (2008), Jupiter's changing auroral location, J. Geophys. Res., 113, A01206, doi:10.1029/2007JA012601.

Guillot, T., D. J. Stevenson, W. B. Hubbard, and D. Saumon (2005), The interior of Jupiter, in Jupiter, edited by F. Bagenal, T. E. Towling, and W. B. Mc Kinnon, pp. 35-37, Cambridge Univ. Press, New York.

Hill, T. W., and A. J. Dessler (1995), Jupiter's magnetosphere, Earth Space, $8(6)$.

Hill, T. W. (2001), The Jovian auroral oval, J. Geophys. Res., 106, $8101-8107$.

Khurana, K. K. (2001), Influence of solar wind on Jupiter's magnetosphere deduced from currents in the equatorial plane, J. Geophys. Res., 106(A11), 25,999-26,016.

Krupp, N., A. Lagg, S. Livi, B. Wilken, J. Woch, E. C. Roelof, and D. J. Williams (2001), Global flows of energetic ions in Jupiter's equatorial plane: First-order approximation, J. Geophys. Res., 106, 26,017-26,032.

Mauk, B. H., D. J. Williams, R. W. McEntire, K. K. Khurana, and J. G. Roederer (1999), Storm-like dynamics of Jupiter's inner and middle magnetosphere, J. Geophys. Res., 104, 22,759-22,778.

Mauk, B. H., J. T. Clarke, D. Grodent, J. H. Waite Jr., C. P. Paranicas, and D. J. Williams (2002), Transient aurora on Jupiter from injections of magnetospheric electrons, Nature, 415, 1003-1005.

Nichols, J. D., and S. W. H. Cowley (2004), Magnetosphere-ionosphere coupling currents in Jupiter's middle magnetosphere: Effect of precipitation-induced enhancement of the ionospheric Pedersen conductivity, Ann. Geophys., 22, 1799-1827.

Prangé, R., S. Maurice, W. M. Harris, D. Rego, and T. Livengood (1997), Comparison of IUE and HST diagnostics of the Jovian Aurorae, J. Geophys. Res., 102(E4), 9289-9301.

Radioti, A., J.-C. Gérard, D. Grodent, B. Bonfond, N. Krupp, and J. Woch (2008), Discontinuity in Jupiter's main auroral oval, J. Geophys. Res., 113, A01215, doi:10.1029/2007JA012610.

Southwood, D. J., and M. G. Kivelson (2001), A new perspective concerning the influence of the solar wind on the Jovian magnetosphere, J. Geophys. Res., 106, 6123-6130

Tomás, A. T., J. Woch, N. Krupp, A. Lagg, K.-H. Glassmeier, and W. S. Kurth (2004), Energetic electrons in the inner part of the Jovian magnetosphere and their relation to auroral emissions, J. Geophys. Res., 109, A06203, doi:10.1029/2004JA010405.

Vasavada, A. R., A. H. Bouchez, A. P. Ingersoll, B. Little, C. D. Anger, and the Galileo SSI Team (1999), Jupiter's visible aurora and Io footprint, J. Geophys. Res., 104, 27,133-27,142.

Vasyliūnas, V. M., and A. J. Dessler (1981), The magnetic-anomaly model of the Jovian magnetosphere: A post-voyager assessment, J. Geophys. Res., 86(A10), 8435-8446.

Woch, J., N. Krupp, and A. Lagg (2002), Particle bursts in the Jovian magnetosphere: Evidence for near-Jupiter neutral line, Geophys. Res. Lett., 29(7), 1138, doi:10.1029/2001GL014080.

B. Bonfond, J.-C. Gérard, D. Grodent, J. Gustin, and A. Radioti, Laboratory for Planetary and Atmospheric Physics, Université de Liège, Allée du 6 Août 17 (B5c), Liége B-4000, Belgium. (d.grodent@ulg.ac.be) J. T. Clarke and J. Nichols, Center for Space Physics, Boston University, 725 Commonwealth Avenue, Boston, MA 02215, USA.

J. E. P. Connerney, NASA Goddard Space Flight Center, Greenbelt, MD 2077, USA. 